\title{
Editorial: New Perspectives in the Philosophy of Physics
}

\author{
Iulian D. Toader ${ }^{1}$
}

Received: 18 May 2015 / Accepted: 26 May 2015 / Published online: 7 June 2015

(C) Springer Science+Business Media New York 2015

This special issue reflects the ongoing close connection between the foundations of physics and philosophy. In the first part, Carsten Held discusses a new argument for the incompleteness of quantum mechanics that does not involve a separation principle, and Elise Crull defends the view that decoherence minimizes any contribution to solving problems in quantum gravity and quantum cosmology that the conflicting interpretations of quantum mechanics may be thought to have. In the second part, Johanna Wolff discusses the role of a priori epistemological principles in articulating and defending alternative metaphysical views about physics, while Elay Shech focuses on idealizations and their potential contribution to the explanation and understanding of certain physical effects. In the third part, Richard Dawid argues that certain limitations of John Norton's material theory of induction are revealed by Norton's dome scenario itself, and Gábor Hofer-Szabó investigates the connection between Bell's local causality and the causal Markov condition, with an emphasis on Bayesian networks.

The idea for this special issue stems from the conference "New Directions in the Philosophy of Physics" and the workshop "Einstein's Philosophy of Science" which took place at the University of Bucharest, Faculty of Philosophy, from 23-25 May 2013. Neither event would have been possible without the encouragement and advice I received from two of my former mentors, Don Howard and Ilie Pârvu, the kind support of the Rector of the University of Bucharest, Mircea Dumitru, as well as the enthusiasm of my diligent graduate students. Half of the articles included in the present issue are revised versions of papers presented in Bucharest. The other half comprises new papers, invited for this special issue. I would like to thank all authors and referees for their efforts, Angela Lahee at Springer, for editorial support, and the editors of this journal, particularly Fedde Benedictus, for inviting me to edit this collection.

$凶 \quad$ Iulian D. Toader

itoad71@gmail.com

1 Department of Theoretical Philosophy, University of Bucharest, Bucharest, Romania 